\title{
Investigation on the Energy Model with Finite Element Method in High Speed Cutting
}

\author{
Lei WAN ${ }^{a}$, Dazhong WANG ${ }^{b}$
}

\author{
College of Mechanical Engineering, Shanghai University of Engineering Science, Shanghai, \\ 201620, China \\ aemail: wanlei789@163.com, bemail:wdzh168@hotmail.com
}

\section{Keywords: Finite Element Method; Energy Model; High Speed Cutting}

\begin{abstract}
This work focused on the energy model of the machining which can be used to predict the energy variation with finite element method. The energy model is validated with the finite element method using the finite element software ABAQUS. The distributions of the stress, strain and temperature as well as the chip morphology are all simulated vividly when cutting stably. It can be found that the variation of energy corresponds to the chip formation and is correlated to distributions of stress and temperature. This study can provide a reference to study the vibration in the high speed machining and optimization of the tool design.
\end{abstract}

\section{Introduction}

Metal cutting can be seen as one of the most complicated machining processes in recent years. For all kinds of metal cutting operations, though the basic mechanics are almost same, each case is considered to be a little different because of the complicity that depends on many factors, such as too edge geometries, material properties, friction model and some variables and so on. With the development of high speed machining, its productivity and quality of its machined surface are becoming increasing attractive to the researchers for industrial developing [1]. While the more distinct part is that the cutting forces will decrease as cutting speed increases and then reaches a relative plateau [2].

For the complexity of the high speed cutting process, analytical and experimental analyses are not suitable to study them. Thanks to the development of computer power and finite element method, simulations of cutting process using commercial FE software become more and more popular for researchers in the past twenty years. Alvarez et al. [3] investigated the effect of different constitutive models on the serrated chip formation, and found that the models have no influence on the chip morphology. Calamaz et al. [4] proposed a new model instead of the JC model to simulate the serrated chip formation, and get a good agreement with the experiments when cutting speed is more than $180 \mathrm{~m} / \mathrm{min}$. Although its popularity, it doesn't simulate the real cutting process completely because the mechanism of cutting process hasn't been grasped completely, and some models should be simplified, and idealizations are necessary. The high speed cutting relates to a lot of parameters, such as strain hardening, rate-dependent hardening and thermal softening, and these main effects can be studied in the speed dependence of chip formation, which is still an advantage of this approach [5].

\section{Energy Model}

To investigate the energy in the cutting process, energy models was proposed by Ma et al. [6]. The total energy expanded in traditional machining can be defined as

$$
U_{a m}=U_{p}+U_{c}
$$

where $U_{p}$ and $U_{c}$ are preheating energy and cutting energy, respectively, as defined in Eqs. (2) and (3)

$$
U_{p}=U_{d}+U_{w}
$$


where $U_{d}$ is the heat energy contained in the depth of cut and $U_{w}$ is the heat energy lost in the work piece.

$$
U_{c}=U_{s}+U_{f}
$$

where $U_{s}$ is the shear energy and $U_{f}$ is friction energy as normally defined in the metal cutting theory.

For clarification, the defined energy in this study concerns only the heat energy absorbed by the workpiece and the mechanical energy caused by the cutting action. As such, some factors on the system scale are not considered which include, for example, the total energy consumption of the tool to support the cutting condition.

\section{Numerical Simulation}

\section{Finite element model}

Fig. 1 shows the simulated cutting process with a finite element model consisted of four parts. A thermo-mechanical coupled analysis is conducted with CPE4RT element type, which was plain strain quadrilateral, linearly interpolated, and thermally coupled elements with reduced integration and automatic hourglass control. In this paper, Lagrangian approach is adopted and workpiece is fixed and cutting speed is applied to the tool, the rake angle is $-10^{\circ}$ and the clearance angle is $3^{\circ}$. The cutting velocity is $2400 \mathrm{~m} / \mathrm{min}$, cutting depth is $1 \mathrm{~mm}$.

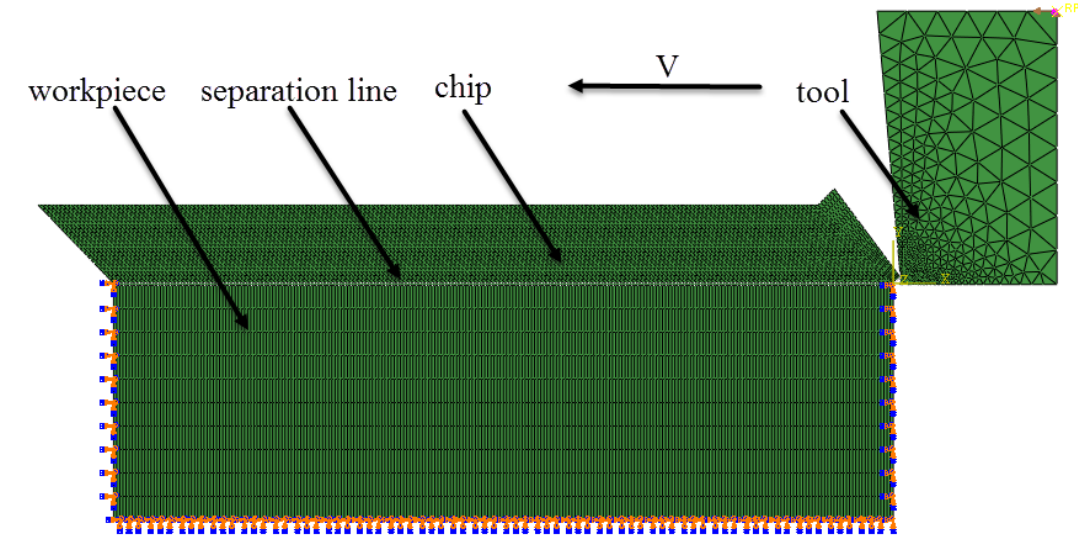

Fig.1 Finite element model

Table 1. Material properties for the workpiece and tool

\begin{tabular}{|l|c|c|}
\hline Material properties & Workpiece & tool \\
\hline Material & AISI 1045 & Carbide tool \\
\hline Young's modulus $(\mathrm{GPa})$ & 200 & 530 \\
\hline Poisson's ratio & 0.3 & 0.13 \\
\hline Thermal conductivity, $\mathrm{k}\left(\mathrm{W} \mathrm{m}{ }^{-1}{ }^{\circ} \mathrm{C}^{-1}\right)$ & 55 & 120 \\
\hline Specific heat $\left(\mathrm{J} \mathrm{kg}^{-1}{ }^{\circ} \mathrm{C}^{-1}\right)$ & 474 & 343.3 \\
\hline Thermal expansion coefficient $\left({ }^{\circ} \mathrm{C}^{-1}\right)$ & $1.4 \times 10^{-6}$ & $5.2 \times 10^{-6}$ \\
\hline
\end{tabular}

\section{Material Constitutive Model}

In finite element models, it is required for a constitutive material model to relate the flow stress to strain, strain rate and temperature to describe the material behavior that is presented with an equation below, which is called JC material model [7].

$$
\sigma=\left(A+B \varepsilon^{\mathrm{n}}\right)\left(1+C \ln \frac{\dot{\varepsilon}}{\dot{\varepsilon_{0}}}\right)\left(1-\left(\frac{T-T_{r}}{T_{m}-T_{r}}\right)^{m}\right)
$$

where $\sigma$ is the equivalent flow stress, $\varepsilon$ the equivalent plastic strain, $\dot{\varepsilon}$ the equivalent plastic strain rate, $\dot{\varepsilon}$ the reference equivalent plastic strain, $\mathrm{T}$ the workpiece temperature, $T_{\mathrm{m}}$ and $T_{\mathrm{r}}$ is 
the temperature of the material melting and room, respectively. $\mathrm{A}, \mathrm{B}, \mathrm{C}, \mathrm{n}$ and $\mathrm{m}$ are constitutive constants. Material properties for the workpiece and tool are presented in the Table 1 [8].

\section{Frictional Model}

When considering machining simulations of AISI 1045, a moderate friction coefficient 0.4 is used. In this study, friction at the tool-chip interface is expressed by the following relations:

$$
\begin{aligned}
\text { in the sliding zone: } & \tau=\mu \sigma_{n}, & \text { if } \mu \sigma_{n}<\bar{m} \frac{\sigma_{0}}{\sqrt{3}} \\
\text { in the sticking zone: } & \tau=\bar{m} \frac{\sigma_{0}}{\sqrt{3},} & \text { if } \quad \mu \sigma_{n}>\bar{m} \frac{\sigma_{0}}{\sqrt{3}}
\end{aligned}
$$

where the $\tau$ is shear stress can be expressed in different forms in the sliding and sticking zones, $\mu$ is the friction coefficient, $\sigma_{n}$ and $\sigma_{0}$ are the normal and the maximum stress, respectively. $\bar{m}$ is a fraction of permissible shear stress of the workpiece material.

\section{Heat transfer model}

Due to plastic deformation in the primary zone and friction behavior at the too-chip interface, heat generation is modeled as a volume heat flux [9]. Heat conduction is assumed to be the primary model of heat transfer, and the equation of heat transfer is as follows:

$$
\int_{V} \rho_{m} \dot{U} d V=\int_{S} q d S+\int_{V} \dot{Q} d V
$$

where $\mathrm{V}$ is the volume of solid material with surface area $\mathrm{S}, \dot{U}$ the material time rate of internal thermal energy, $\rho_{m}$ the mass density, q the heat flux per unit area of the body flowing into the body, and $\dot{Q}$ the heat supplied externally into the body per unit volume.

\section{Result and discussion}

\section{Chip Morphology and Stress Analysis}
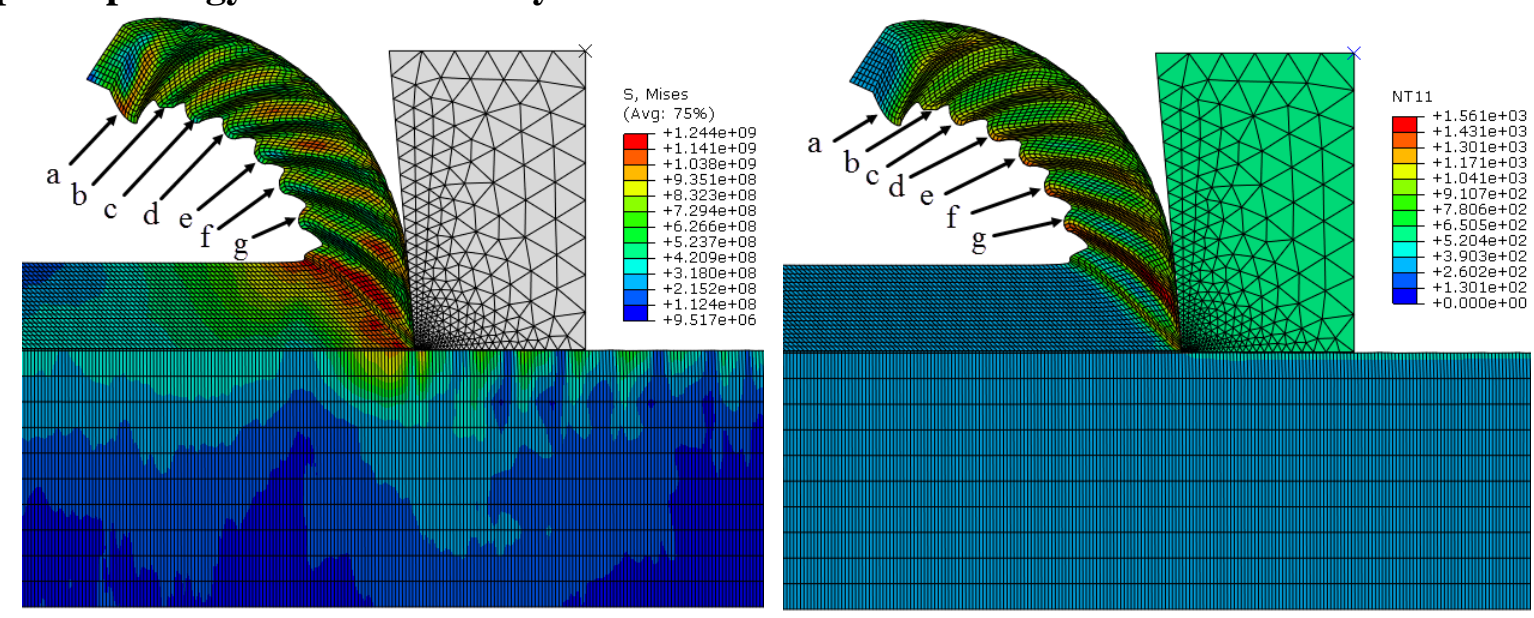

Fig. 2 The distributions of the stress and temperature during the serrated chip formation

Fig.2 shows the finished simulated chip morphology. It can be found in this figure that when serration a forms, the meshes attached to the shearing band in primary zone distorts greatly so that it is easy to be interrupted during the simulation of the cutting process. Also, the stress in the shearing zone is the largest in the workpiece during the cutting process because of the large deformation according to the JC material model before. As the tool advances, the serrated chip formation continues. The end of the second serrated chip $b$ formation is the beginning of the third $\mathrm{c}$ just like the end of the third serrated chip $\mathrm{c}$ is the beginning of the fourth $\mathrm{d}$. It is very interesting to find that several adiabatic shearing bands exist between the adjacent serrations as well as the less stress in the region because of the thermal soften effect which can be found in the right of Fig. 2. The higher temperature in the adiabatic shear band is seen in the figure and its effect of softening the material as well as diminishing the effective stress is presented in this picture. Until the seventh serration ends, it can be found that the shapes of serration are rather regular, and there are clear adiabatic 
shearing bands between these serrations, and the shearing angles are almost same during serrated chip formation.

\section{Cutting force analysis}

Fig. 3 shows the simulated cutting force during the serrated chip formation in high speed cutting process. The figure suggests that the mean value of cutting force is about $620 \mathrm{~N}$ when cutting steadily. But it is also more obvious that the real-time cutting force fluctuates greatly and presents high frequency, periodically, making it harder to detect the change of cutting force by dynamometer because of itself low frequency. The fluctuation between the two points suggests that the formation of serrated chip is not regular, and the reality is the fluctuation should be much more and higher frequency considering the vibration of the machinery and tool as well as the cutting process. The point 1-7 points correspond to the formation of serrations a-g, indicating the real-time value of these forces when cutting. It also can be found that these forces are not equal because of the organization structure inner workpiece material and the vibration in cutting process.

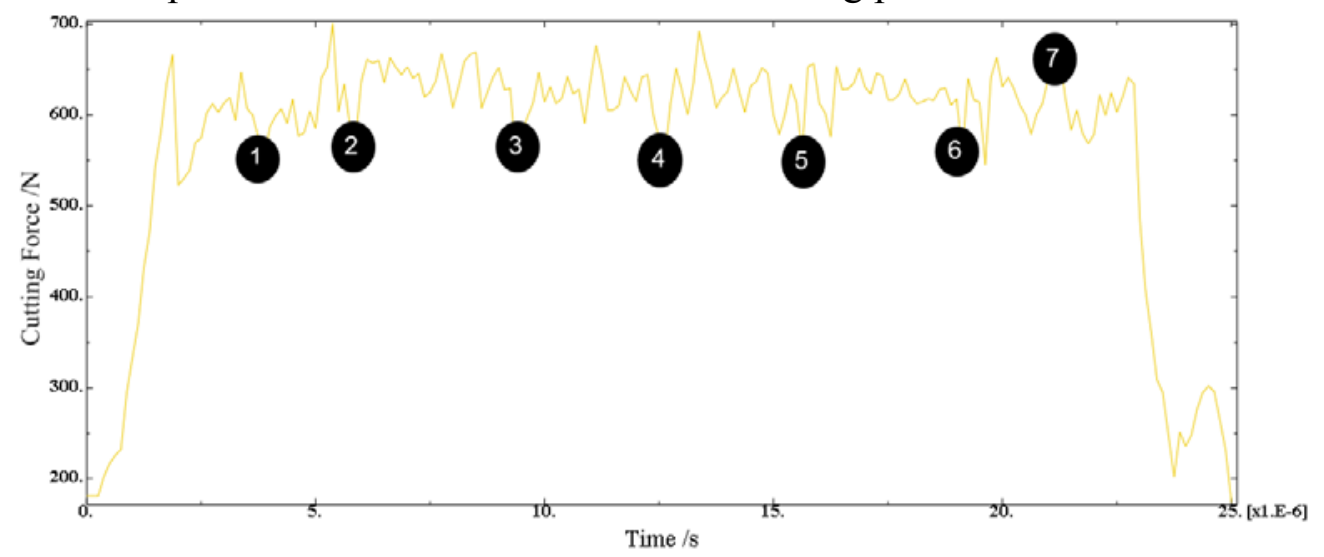

Fig. 3 The variation of the cutting force during the serrated chips (a-g) formation Temperature and strain analysis
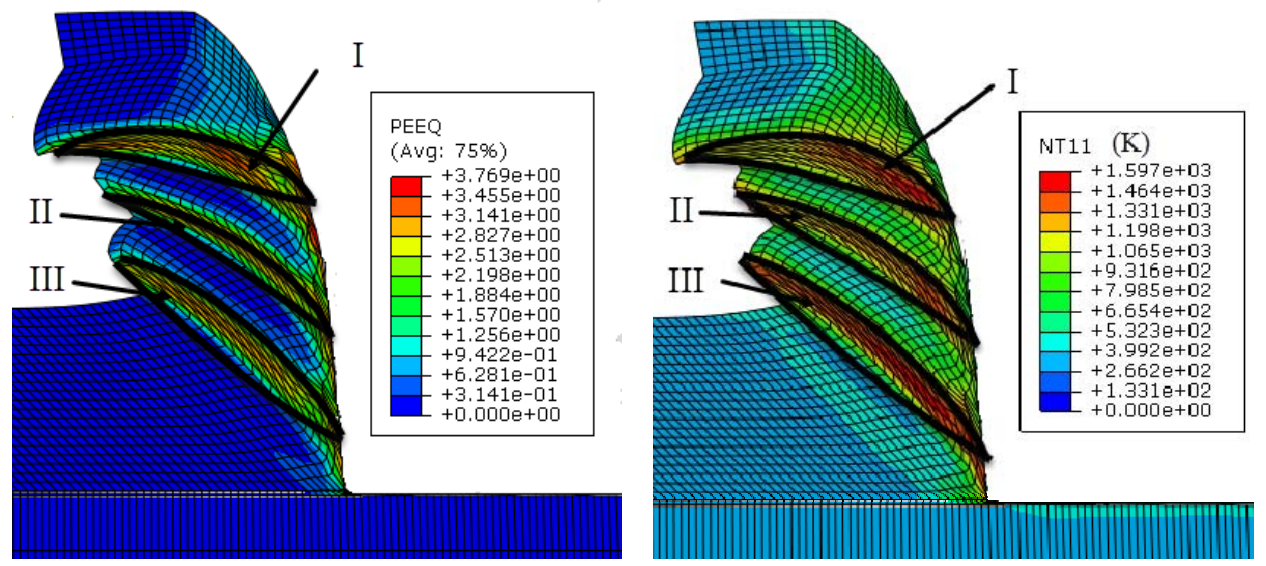

Fig. 4 The distributions of the effective strain and temperature when the serrations I II III formed

It can be seen in Fig. 4 that the value of strain is highest in the primary shearing zone and the region of the chip closed to tool rank face, which can also indicates that the temperature in these zones are also highest. It can be seen in the figure that the high temperature zones include adiabatic shearing bandings (I, II, III) and region in front of tool rank face besides primary shearing zone. The highest temperature reaches $1500 \mathrm{~K}$ because of the high strain of metal material in primary shearing zone, and the temperature in adiabatic shearing band also reaches a relatively high for the heat does not transfer out just in time. The high temperature zone in front of tool rank face is caused by friction between tool and chip and high strain which is shown in Fig. 4. It also can be found that temperatures of zones between the adiabatic shearing bands are not too much high, because their heat sources all come from the heat transition, but the time is too short.

\section{Total energy analysis}

Fig. 5 shows the variation of the total energy during the serrated chips (a-g) formation using finite element method in high speed cutting. The peaks on the curve from A to $G$ correspond to the 
serrated chips a-g. During every serrated chip forming, there always exists a peak and a valley as well as the same curve. It can be seen in the Fig. 5 that after cutting steadily, the total energy increases all the same, but decreases after every serrated chip formation due to the formation of the adiabatic shearing band, and the heat cannot transfers out, thus making the material soften, and the material flow start to slow down. But in general, the consuming of the total energy still increases as the cutting process goes on.

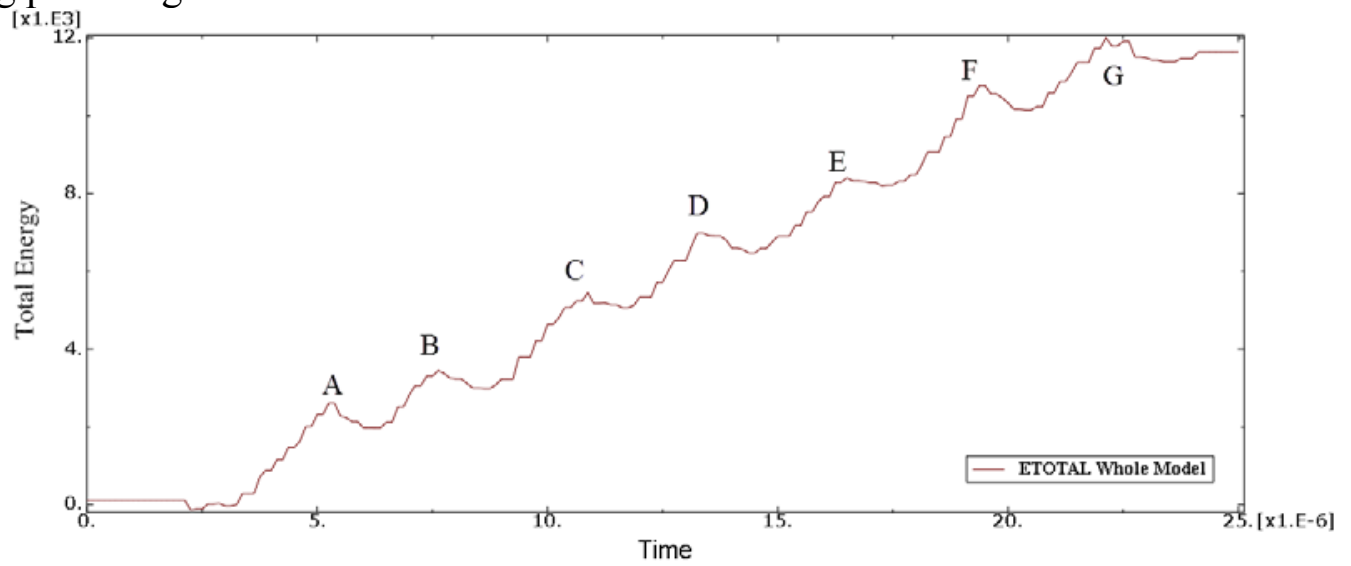

\section{Conclusions}

Fig. 5. The total energy during the serrated chip formation in high speed cutting process

This paper simulated the formation of serrated chip with ABAQUS/Explicit and obtained many useful data. This phenomenon is based on the energy model and explained by adiabatic shearing theory. Extra works can be done to study the cutting feature with different tools and workpiece materials under different cutting conditions to provide reference for tool designing or optimization of cutting parameters, for the purpose of optimizing the cutting process and least production cost.

\section{Reference}

[1] F.W. Bach and H. Schulz, Scientific fundamentals of HSC: Hanser, 2001.

[2] W. Konig, R. Lowin, K. Steffens, and V. Hann, Aspekte zur Technologie der Hochgeschwindigkeitszerspanung, Industrieanzeiger, 1981 (103) 14-20.

[3]R. Alvarez, R. Domingo, and M. A. Sebastian, The Formation of Saw Toothed Chip in a Titanium Alloy: Influence of Constitutive Models, Strojniški vestnik - Journal of Mechanical Engineering, 2011 (57) 739-749.

[4] M. Calamaz, D. Coupard, and F. Girot, A new material model for 2D numerical simulation of serrated chip formation when machining titanium alloy $\mathrm{Ti}-6 \mathrm{Al}-4 \mathrm{~V}$, International Journal of Machine Tools and Manufacture, 2008 (48) 275-288.

[5] M. Bäker, Finite element simulation of high-speed cutting forces, Journal of Materials Processing Technology, 2006 (176) 117-126.

[6] J. Ma, X. Ge, and S. Lei, Energy efficiency in thermally assisted machining of titanium alloy: A numerical study, Journal of Manufacturing Science and Engineering, 2013 (135).

[7] G. R. Johnson and W. H. Cook, A constitutive model and data for metals subjected to large strains, high strain rates and high temperatures, in Proceedings of the 7th International Symposium on Ballistics, 1983 541-547.

[8] T. Kagnaya, L. Lambert, M. Lazard, C. Boher, and T. Cutard, Investigation and FEA-based simulation of tool wear geometry and metal oxide effect on cutting process variables, Simulation Modelling Practice and Theory, 2014(42) 84-97.

[9] K. Liu and S. N. Melkote, Finite element analysis of the influence of tool edge radius on size effect in orthogonal micro-cutting process, International Journal of Mechanical Sciences, 2007 (49) 650-660. 